\title{
El cuerpo del Héroe: el descubrimiento del busto de un Soldado caído en la Guerra de Malvinas
}

\author{
Laura Marina Panizo* \\ ${ }^{*}$ CONICET, Instituto de Altos Estudios Sociales, Universidad Nacional de \\ General San Martín. E-mail: laura.m.panizo@gmail.com
}

RMA

Antropología Social

\begin{abstract}
Resumen
En este artículo veremos de qué manera el descubrimiento del busto del soldado caído en la Guerra de Malvinas, Elbio Araujo, expresa un marco simbólico de interpretación de la muerte en la guerra de un grupo particular, y deviene para los familiares en un escenario de dramatización de la muerte que por la falta del cuerpo no pudo enfrentarse a través de sus formas habituales. Observaré al ritual desde el punto de vista de la performance, ya que nos permite ver de qué manera estamos refiriéndonos a experiencias vivenciadas en escenarios determinados, a través de los cuales es habitada la muerte de una persona, y significada de una manera particular. Así, veremos que a través del descubrimiento del busto, se sustituye la presencia del cuerpo ausente por otra materialidad y la puesta en escena actúa no sólo como un canal de comunicación a través del cual los deudos expresan al resto de la sociedad sus experiencias y los sentidos dados a la muerte en la guerra, sino que deviene también, para los familiares involucrados, en una práctica transformadora en cuanto a la reflexividad que propone sobre la condición social del muerto.
\end{abstract}

Palabras clave: performance; guerra; duelo; Malvinas.

The Hero's Body: the Unveiling of the Bust of a Soldier Fallen in the Malvinas War

\begin{abstract}
In this paper we will show how the unveiling of the bust of Elbio Araujo, a soldier fallen in the Malvinas War, expresses the symbolic framework for interpretation of death in battle of a particular group, at the same time becoming for his relatives the stage for dramatization of a death that could not be dealt with in the usual manner due to the lack of a body. We will deal with the ritual from the point of view of performance, since it allows us to see the ways experiences are lived in specific scenes, through which the death of a person is inhabited and signified in a particular way. Therefore, we will show that through the unveiling of the bust, the lack of the body is replaced by a different materiality and that the performance acts not only as a communication channel through which the bereaved express their experiences and the meanings given to death in war to society at large, but also becomes, for the families involved, a transformative practice through the proposed reflexivity on the social status of the deceased.
\end{abstract}

Keywords: performance, war, mourning, Malvinas.

Tanto Philippe Aries (1999) como otros autores (Sthephens, 2007; Grant 2004), hacen hincapié en la ruptura que se produce después de la Primera Guerra Mundial en las actitudes tradicionales frente a la muerte. La matanza de gente en una dimensión sin precedentes, puso de manifiesto la insuficiencia de las prácticas mortuorias y conmemoraciones tradicionales, y forzaron a una clase de prácticas conmemorativas a través de la construcción de monumentos y cementerios, que combinaron el culto a los muertos y el sentimiento nacional. En su artículo acerca de la construcción del monumento de Victoria Park, en Perth, al oeste de Australia, John Sthephens propone explorar las características entre la materialidad del monumento y su significado para la comunidad y los núcleos que conectan objetos y memoria en el contexto de los monumentos de guerra. El autor señala sobre los monumentos australianos erigidos en conmemoración de los muertos de la Segunda Guerra Mundial, que entre sus múltiples efectos le da a los familiares la posibilidad de "una expresión concreta de su memoria, su pérdida y el dolor de la ausencia" (2007:242) ${ }^{1 *}$ de sus seres queridos, así como hacer referencia al sacrificio nacional. Así también, Jacques Le Goff, refiere que entre las manifestaciones significativas de la memoria colectiva se puede citar la aparición después de la Primera Guerra Mundial, del monumento al Soldado Desconocido con el objeto de proclamar

\footnotetext{
${ }^{1}$ De aquí en mas el asterisco significa mi propia traducción del inglés.
} 
"con el cadáver sin nombre la cohesión de la nación a la memoria común" (1991:172). Con el mismo sentido, en Argentina se erigieron en diversas ciudades cenotafios y monumentos en memoria de los combatientes caídos en la Guerra de Malvinas. Se denomina comúnmente Guerra de Malvinas al conflicto bélico entre Argentina y Gran Bretaña entre 2 de abril y el 14 de junio de 1982. Esta guerra se dio en el contexto de un gobierno dictatorial que tomó el poder en 1976 y que fue responsable de la detención clandestina y desaparición física de miles de ciudadanos. En el año 1982 el gobierno militar decide recuperar las Islas Malvinas a la fuerza, lo que llevo a un conflicto bélico con Gran Bretaña dejando un saldo de 469 muertos argentinos. De las 469 víctimas en el Cementerio de Darwin hay sólo 237 sepultadas en 230 tumbas individuales, y dos fosas comunes: una con 4 tripulantes de un helicóptero y otra con 3 tripulantes de un Lear Jet derribado. De las 230 tumbas, 123 poseen lápidas con la leyenda "Soldado sólo conocido por Dios" y 107, incluyendo las dos fosas comunes, poseen lápidas con los nombres y apellidos de los sepultados. No yacen en el cementerio los 323 muertos víctimas del hundimiento del Crucero General Belgrano, 2 de los tripulantes del Lear Jet que están sepultados en la isla Borbón, varios caídos que se hundieron en otros buques, un número de pilotos derribados y algunos que fueron enterrados en la Argentina continental.

Como había mencionado, en Argentina se erigieron en diversas ciudades cenotafios y monumentos en memoria de los combatientes caídos en la Guerra de Malvinas. El primer cenotafio destinado a Malvinas promovido por el Estado Nacional, fue construido en el segundo gobierno de Carlos Saúl Menem, situado al pie de la barranca de la Plaza San Martín que da a la Avenida del Libertador, en Buenos Aires, y está formado por 25 placas de mármol negro con los nombres de los 649 combatientes caídos en la guerra². Entre todos los cenotafios y monumentos en memoria de los muertos en la Guerra, es el Monumento en el cementerio de Darwin, Islas Soledad, el que tiene la peculiaridad de que, promovido y gestionado por la comisión de Familiares de caídos en la Guerra de Malvinas e Islas del Atlántico Sur ${ }^{3}$, yace en el lugar donde no solo están los cuerpos recuperados de los caídos en Malvinas, sino en el territorio en disputa que motivó esa guerra que culminó con la derrota Argentina, y que está a la espera de su recuperación nacional.

Este cenotafio, que fue donado por el empresario Eduardo Eurnekian, fue promovido por Familiares de Malvinas durante 25 años y finalmente llevado a las islas en el año 2004. La construcción del Monumento en Darwin se presenta como un hecho inédito en la historia de enfrentamientos bélicos, en tanto pertenece al pueblo

\footnotetext{
2 Para profundizar en la construcción, y la inauguración del monumento, y en los debates que rodearon dicha construcción consultar, Guber (2004:169-184).

3 De aquí en más me referiré a este organismo como Familiares de Malvinas.
}

derrotado, pero es administrado por el vencedor:

"Los propios británicos definen a la construcción del Monumento a los Soldados Argentinos Caídos en Malvinas e Islas del Atlántico Sur como un hecho inédito y extraordinario en la historia universal. Nunca antes se había logrado que dos naciones que sostuvieron una guerra por un territorio, subsistiendo las razones de esa conflagración -la disputa por la soberanía -, pudieran ponerse de acuerdo en permitir la construcción de un Monumento en memoria de los soldados Caídos, pertenecientes al ejército derrotado por la vía armada, en el propio territorio en disputa, y que se encuentra bajo administración del ejército vencedor". ${ }^{4}$ Así, refieren los familiares respecto al cementerio como "la única presencia permanente de los argentinos en nuestras islas durante la postguerra"5.

Los significados que el cenotafio en Darwin condensa, van más allá de la conmemoración a una relación (familiares y muertos) sino que invoca, tal como lo sostiene Grant (2004:81), acerca de los marcadores de mármol sobre la tumba de la Guerra Civil Americana, una relación entre los soldados y una Nación, la tierra, que en el caso de Malvinas se entiende y pretende como nacional (Panizo 2011:163) ${ }^{6}$.

En el caso de la Guerra de Malvinas, como en las guerras en general, no todos los cuerpos fueron identificados. Así también, los cuerpos identificados yacen como habíamos mencionado, en el cementerio de Darwin. En este sentido, la muerte en la guerra y la falta del cuerpo produjo en los familiares una ruptura en las formas habituales de enfrentar la muerte por lo que la construcción de monumentos, y memoriales, al igual que en los casos recientemente citados, devinieron en prácticas mortuorias y conmemorativas, que no solo tienen el objeto de hacer culto a los muertos sino de dar un sentido específico al conflicto bélico determinado. La construcción de los bustos y estatuas también cumplen estas funciones con la diferencia que simbolizan el cuerpo de una persona específica, individualizando la muerte, el homenaje, frente a los monumentos que proponen una memoria común a los muertos en plural. En la argentina se construyeron diversos bustos de militares y excepcionalmente de soldados de la guerra. En este artículo analizaremos el descubrimiento del Busto del Soldado Elbio Araujo, cuyos familiares forman parte del organismo Familiares de Malvinas. Como veremos, este evento funcionó como un ritual mortuorio que reactualizó el acontecimiento de la muerte del caído y permitió se expresaran los sentidos dados a la muerte en la guerra por parte de los familiares y la comisión en la arena pública.

\footnotetext{
4 Comisión de Familiares de Caídos en Malvinas e Islas del Atlántico Sur, en http://www.noretornable.com.ar/v2/dossier/carta.html

5 Comisión de Familiares de Caídos en Malvinas e Islas del Atlántico Sur, en http://www.noretornable.com.ar/v2/dossier/carta.html

6 Para profundizar sobre la inauguración del cenotafio ver Panizo, 2011:162-173.
} 
Para entender el significado que la familia Araujo le dio a la construcción e inauguración del busto debemos primero considerar que ellos se adaptaron a los cambios para sobrellevar la pérdida de su ser querido integrándose a la comisión de Familiares de Malvinas en la posguerra. Esta comisión, funciona como un grupo que, en tanto comunidad de pares que pasaron por experiencias similares, cumple un rol fundamental en cuanto le da a sus miembros herramientas para enfrentar las pérdidas, suministrando también un fuerte sentimiento de identidad e integración social ${ }^{7}$. Al igual que los llamados grupos de autoayuda o ayuda mutua, en los cuales los individuos se apoyan entre sí para sobrellevar sus experiencias estresantes o traumáticas acontecidas por una problemática en común, como el caso de enfermedades, adicciones, estigmatización social, etc., los familiares de los muertos en Malvinas se sienten, dentro de su institución, comprendidos por personas que atravesaron la experiencia de la muerte de un ser querido en la guerra. Pero a diferencia de los grupos de autoayuda, en Familiares de Malvinas, la memoria y la ideología del grupo aparecen como componentes esenciales, en tanto la temática referida a la guerra es reelaborada según ciertos parámetros de identidad grupal que obedecen a los proyectos específicos del grupo. Así, la integración del individuo al grupo ha permitido un proceso de homogenización que hace a la identidad grupal, neutraliza las diferencias personales y permite una narrativa y un entendimiento común sobre la muerte en la guerra. En este sentido, hablaré de marco simbólico de interpretación, para dar cuenta del repertorio simbólico a través del cual los familiares le dan sentido a la muerte en la guerra.

El concepto de marco, que fue legitimado en el ámbito de las ciencias sociales por Erving Goffman (1974), hace referencia, según el autor, a los principios de entendimiento que organizan la experiencia cotidiana del individuo y orientan la acción. Son, en este sentido, un conjunto de orientaciones mentales que permiten interpretar hechos sociales significativos. Varios autores adoptaron los aportes de Goffman en los estudios de los movimientos sociales para designar esquemas de interpretación definiéndose por su función orientadora y organizadora de la experiencia (Carozzi, 1998:21). En este sentido, ha sido utilizado por varios investigadores que trabajan con movimientos religiosos para referirse a "la reproducción de situaciones sistemáticamente transformadas", que modifican los esquemas de entendimiento previos (Ibíd.).

Por otro lado, fue el sociólogo Maurice Halbwachs (2005), de la escuela Durkhemiana, quien introdujo, en el campo de los estudios sobre memoria, la idea de marcos sociales para hablar de la memoria colectiva, y plantea una interrelación entre memoria individual y

\footnotetext{
7 Michael Pollak (2006) y Elizabeth Jelin (2002) trabajan la importancia de la integración de individuos que han vivido experiencias traumáticas en comunidades de iguales, donde se refuerzan los sentimientos de identidad, continuidad y unidad.
}

memoria colectiva al proponer la idea de que el grupo y la sociedad son las condiciones, los marcos sociales de la memoria. A la noción de memoria colectiva se suma la de "corriente de pensamiento", que nos relaciona con un grupo determinado, por lo que ambas aparecen enmarcando a los grupos de referencia. En este punto, Halbwachs resalta la importancia de los intereses del presente y las condiciones sociales que hacen posible la conciencia de cada uno. Desde la sociología, también Michael Pollak (2006) retoma las cuestiones planteadas por Halbwachs y aporta elementos para introducir la carga conflictiva en las visiones acerca de cómo recordar el pasado en el presente. Dentro de este marco, Pollak ha dedicado buena parte de sus estudios a las experiencias humanas frente a situaciones límite y memoria, tomando como eje dos tipos de situaciones divergentes: por un lado, el genocidio nazi y, por el otro, la epidemia del sida. Sus trabajos han servido como modelo para estudiar las memorias en el Cono Sur, marcadas por procesos de violencia política y represión estatal en el marco de dictaduras militares. El autor plantea una estrecha relación entre memoria y sentimiento de identidad, al entender a la memoria como una operación colectiva que intenta definir y reforzar sentimientos de pertenencia y fronteras sociales (Pollak 2006:38). Cada forma de interpretar el pasado y el presente por un grupo social, puede ser entendida, en términos de este autor, como un proceso de construcción de una memoria colectiva, la memoria política del grupo que, a través de un trabajo de encuadramiento y mantenimiento, dan a cada miembro el sentimiento de unidad, coherencia y continuidad. De esta manera, la memoria se torna en un elemento constituyente de identidad. Dentro de este marco, Elizabeth Jelin (2002) señala que el individuo, para fijar parámetros de identidad, selecciona ciertos hitos que lo ponen en relación con otros y constituyen los marcos sociales para encuadrar las memorias (Ibíd.: 25). También la autora nos llama la atención sobre el hecho de que estas interpretaciones y memorias del pasado reciente aparecen en un escenario de luchas políticas acerca del sentido, no sólo de lo ocurrido, sino también de la memoria misma. De esta manera, el espacio de la memoria deviene en un espacio de lucha política que puede ser entendida en términos de las luchas de la "memoria contra memoria" (lbíd.:6).

Todos estos trabajos resultaron muy ilustrativos para pensar la forma en que los familiares significan la muerte de acuerdo con la forma en que le dan sentido al pasado y se identifican dentro de un grupo. En este sentido, sumándome a estos estudios, retomaré los aportes de los trabajos sobre movimientos sociales, que han usado el concepto de marco interpretativo para designar esquemas de interpretación, que se definen por su función orientadora y organizadora de la experiencia (Carozzi, 1998:33). Así, alineándome con los trabajos de Maria Julia Carozzi que define los marcos de movimientos sociales y religiosos por su función transformadora de los esquemas interpretativos previos (Ibíd:: 37) he llamado marco 
simbólico de interpretación al modelo de interpretación del grupo de Familiares de Malvinas que, a través de una selección de símbolos y una ideología particular orienta la forma en que los familiares deben darle sentido a la muerte en el contexto de la guerra (Panizo 2011:7). Hablo de marco simbólicos de interpretación, ya que se pondrá énfasis en que la manera en que los familiares entienden el pasado y el presente, como modo de interpretación de la realidad, forma parte de un contexto cultural general en donde "los modos de recordar" se articulan con procesos de apropiación y reelaboración de símbolos y metáforas históricamente construidas. Así, las "memorias", acciones políticas, rituales sociales, rituales mortuorios, prácticas públicas y domésticas, se alimentan recíprocamente y están representadas en símbolos a través de los cuales los familiares actúan y se movilizan.

Las representaciones que se generan en este marco de interpretación responden a representaciones, valores y prácticas estructuradas a escala nacional. Muchas de estas configuraciones culturales, como la categoría de héroe, o los distintivos nacionales, devienen en herramientas culturales que expresan y dramatizan una diversidad de significados a través de los cuales los familiares entienden, no sólo la relación con sus parientes muertos, sino también con la historia cultural.

Entonces, los marcos simbólicos del grupo son repertorios simbólicos que orientan las prácticas y significaciones de los familiares para responder a la problemática de la muerte de acuerdo con los intereses de los grupos. Es a través de estos modelos que los familiares simbolizan un tipo de relación específica con sus seres queridos, y se identifican entre sí como miembros de un grupo social determinado. En este sentido, el análisis de los rituales mortuorios o de las prácticas que hacen referencia a la muerte nos permite entender la forma en que los familiares simbolizan las experiencias límite vividas. Estas prácticas, entonces, son entendidas no sólo como instancias de despedida, separación e integración de los sujetos sociales (tanto vivos como muertos), sino como medios expresivos de comunicación y reflexividad que dan cuenta del marco social a través del cual los individuos les dan sentido al pasado y al presente y se identifican dentro de un grupo.

A continuación, veremos de qué manera Familiares de Malvinas construyeron un marco simbólico de interpretación de lo ocurrido que, haciendo referencia a la mitología heroica nacional oficial, puso énfasis en la "argentinidad" como concepto que engloba a una nación unificada por los valores, creencias y prácticas religiosas católicas que ellos entienden como tradicionales y características del pueblo argentino.

\section{Comisión de Familiares de Caídos en Malvinas e Islas del Atlántico Sur.}

Familiares de Malvinas se origina por un grupo de familiares que se empezaron a reunir una vez culminada la guerra, por la necesidad de encontrarse con pares que habían sufrido las pérdidas de sus seres queridos en ésta, las necesidades de honrar a los muertos, conocer las Islas Malvinas y construir un monumento en el cementerio de Darwin, Isla Soledad. Así, conforman la institución básicamente hermanos, padres y madres de caídos, ya sean soldados conscriptos o personal de cuadro, de las tres fuerzas (Fuerza Aérea, Armada, y Ejercito).

Se constituye entonces como comisión en el año 1994 en Capital Federal, con los propósitos de honrar públicamente a sus seres queridos muertos en la Guerra, de reafirmar los derechos soberanos sobre las Islas Malvinas, y de promulgar actividades que entre otras cosas, reafirmen, en sus palabras, el "concepto de argentinidad" y "afiancen los valores culturales, espirituales y sociales que caracterizan al pueblo argentino". Así, dentro del entramado simbólico de la historia argentina, de aquellos símbolos asociados a la unificación de la diversidad cultural como los patrios, los religiosos, y las figuras de los próceres, los familiares de Malvinas reivindican el compromiso con la bandera, se reapropian de la imagen de la Virgen de Lujan ${ }^{8}$, e identifican a sus familiares con los héroes nacionales consagrados por la historia oficial. Así también, frente a las representaciones asociadas a la historia reciente, donde la victimización y la denuncia de violación a los Derechos Humanos está asociada a la desaparición de personas, la elaboración de sentido de la muerte de los caídos en la guerra, enmarcada también en el contexto de la última dictadura militar, fue sustentado recurriendo a la idea de la unidad nacional. Haciendo entonces referencia a la mitología heroica nacional oficial, este organismo pone énfasis en la "argentinidad" como concepto que engloba a una nación unificada por los valores, creencias y prácticas religiosas católicas que ellos entienden como tradicionales y características del pueblo argentino. Podemos hablar así de un repertorio simbólico que orienta las prácticas y significaciones de los familiares para responder a la problemática de la muerte de acuerdo con los intereses del grupo. Es a través de este repertorio, más o menos hegemónico, que los familiares simbolizan un tipo de relación específica con sus seres queridos, y se identifican entre sí como miembros de un grupo social determinado. Este marco, con sus parámetros e hitos de identidad específicos, va recreándose constantemente, para adecuarse a las particularidades de los momentos históricos vividos ${ }^{9}$.

\footnotetext{
8 En todas las actividades organizadas por la Comisión de Familiares, la Virgen de Luján ha jugado un rol fundamental, en cuanto por un lado deviene en símbolo dominante en la acción ritual, y por el otro, condensa múltiples significados que hacen referencia a la guerra, la nación, el patriotismo, la protección y la maternidad. Para profundizar sobre este tema ver Panizo 2011:134-145.

9 En este sentido es importante resaltar que Héctor Cisneros presidente de la comisión durante 27 años consecutivos jugó un rol significativo en la construcción de este marco de interpretación. Sin embargo, renuncia a su cargo en el año 2010, luego de que el matutino Crítica de la Argentina publicara una nota en la que se dio a conocer que su nombre figuraba en el listado de los miembros del Batallón 601, el
} 
De la puesta en marcha del objetivo primordial de Familiares de honrar públicamente a los héroes, se van llevando a cabo algunas actividades que terminan funcionando como espacios sociales en donde no sólo se presentan a los caídos de la guerra como héroes nacionales sino que devienen en rituales o espacios mortuorios donde los deudos se enfrentan a la muerte "cara a cara" en espacios de atención ritual ${ }^{10}$. Esto resulta relevante para los familiares ya que como habíamos mencionado, se enfrentan al hecho de la falta del cuerpo y la imposibilidad de realizar cualquier ritual mortuorio convencional. En este sentido, los familiares manifiestan las dificultades que tuvieron para afrontar la muerte, ya que las prácticas rituales habituales se vieron impedidas:

"La desaparición física de una persona es terrible porque, vos, tenés que aceptar la muerte sin verlo, sin saber si es cierto, por más que te digan, que te cuenten y demás, siempre, te queda la duda" (Nilda, hermana de un caído en Malvinas, 28/10/09).

Para sortear los obstáculos y las ambigüedades que produce la falta del cuerpo, para los familiares fue importante entender las pérdidas no sólo tomando los recursos que propone el Estado a través de, por ejemplo, las notificaciones oficiales amparadas en la Ley de Ausencia con Presunción de Fallecimiento (Ley 14.394) ${ }^{11}$, sino también los de la historia nacional. Así, como había mencionado, los familiares enfatizan en la figura del héroe, intentan abstraer la guerra del Terrorismo de Estado y propone incluir a los caídos en la guerra en el panteón de referencia de los grandes ciudadanos nacionales. De este modo, los héroes de Malvinas,

organismo de Inteligencia del Ejército durante la dictadura. Allí se lo acusaba de haberse desempeñado como analista de información de esa central de espionaje durante el Proceso. Por ello, Familiares de Malvinas aceptó la renuncia de su titular, ocupando este cargo Delmira de Cao. El impacto que la renuncia de Héctor Cisneros tuvo en la Comisión no fue trabajada por ser posterior al trabajo de campo realizado en mi investigación, sin embargo considero relevante su profundización en el futuro.

10 Uso el termino de "atención ritual" para diferenciar esta forma de enfrentamiento a la muerte con aquella que hemos llamado "muerte desatendida", en el caso de los desaparecidos de la última dictadura militar, en donde por la falta del cuerpo, no hay un enfrentamiento claro a la muerte y no se generan espacios de acción ritual, en donde tanto los muertos como los deudos sean atendidos por parte de la sociedad. Para profundizar sobre esto, ver Panizo, 2009.

11 Dicha ley determina la presunción de fallecimiento a la desaparición de cualquier persona domiciliada o residente en el país, de la cual no se tenga noticia por el término tres años. Se presume también el fallecimiento de un ausente: "1) Cuando se hubiese encontrado en el lugar de un incendio, terremoto, acción de guerra u otro suceso semejante, susceptible de ocasionar la muerte, o hubiere participado en una empresa que implique el mismo riesgo y que no se tuviere noticias de él por el término de dos años contados desde el día en que ocurrió, o pudo haber ocurrido el suceso; 2) Si encontrándose en una nave o aeronave naufragada o perdida, no se tuviere noticia de su existencia por el término de seis meses desde el día en que el suceso ocurrió o pudo haber ocurrido" (artículo 23, Ley 14.394). En el caso de los desaparecidos, la Ley de Ausencia por Desaparición Forzada reemplaza a la ley de recién mencionada, para que los familiares puedan hacer trámites legales sin tener que suponer muerto al desaparecido. Para profundizar sobre esto consultar Panizo, 2009. ubicados a la par de otros héroes nacionales, se distinguen de otros muertos, como los desaparecidos, ya que según lo entienden ellos, no murieron en defensa de un territorio nacional amenazado por fuerzas extranjeras:

"Aparte, la diferencia que tenemos con Hebe de Bonafini, es que sus hijos se murieron, supuestamente... peleando contra los propios hermanos. A mi hermano no lo mató un militar, a mi hermano lo mató una bomba inglesa y mi hermano muere defendiendo a los subversivos, a los militares, a los radicales, a los peronistas, a los católicos, a los judíos, a todos los que viven en esta patria. Mi hermano dio su sangre por todo. Esa es la gran diferencia, que la muerte de ellos haya valido y que sea valiosa porque fue más allá de la dictadura" (María Fernanda, hermana de un caído en Malvinas, 03/04/09).

"Murió por la patria, lo llevaron legalmente, no los llevaron ocultos, esa es la diferencia entre los desaparecidos y los nuestros. Un pueblo aplaudió, el 2 de abril se llenó la plaza ¿Por qué no se puso el pueblo en contra?" (Delmira, madre un caído en Malvinas, 03/09/09).

Con la idea de que los caídos dejaron su sangre en un territorio de todos, por una causa nacional, los Familiares utilizan una metáfora orgánica para entender a la nación como una comunidad moral, que va más allá de sus gobernantes. Esta asociación simbólica cuerpo-nación, presente en las representaciones de todos los familiares, legitima la incorporación de los caídos en Malvinas, al linaje de los ciudadanos ilustres nacionales (Guber, 2001; Lorenz, 2006). Dado entonces, que sus seres queridos dieron su vida por la Patria y fueron consagrados a la vez por el Estado argentino como héroes nacionales a través de la ley Nacional $24.950^{12}$, el objetivo principal de Familiares de Malvinas fue, desde sus inicios, preservar la memoria de los caídos en tanto héroes nacionales y promover una actitud social de honrarlos públicamente, como ha sucedido con otros muertos ilustres que lo antecedieron.

La categoría de héroe remite, en estos casos, a vidas trágicamente interrumpidas por una causa nacional, y que por lo tanto, son consideradas merecedoras de un peculiar reconocimiento social. Como señala Santiago Álvarez, las muertes violentas, cuando son consideradas altruistas pueden elevar a la persona a la categoría de Héroe (2001:31). En este caso, el acto heroico enmarcado en un repertorio que podemos denominar nacionalista ${ }^{13}$,

\footnotetext{
12 La ley N²4.950, promulgada el 3 de abril de 1998, declara Héroes Nacionales a los combatientes argentinos fallecidos en defensa de la soberanía nacional sobre las islas del Atlántico Sur, en el conflicto de 1982.

13 Aunque no ignoro la heterogeneidad de ideas acerca de la Nación, y la diversidad de formas en que diferentes grupos sociales pueden entenderse como nacionalistas, entiendo esta forma de entender la muerte como nacionalista en cuanto ellos enfatizan sobre la idea de nación como una comunidad que unifica a los ciudadanos a través de diferentes símbolos y prácticas culturales a escala nacional, que los diferencian con los extranjeros.
} 
implica para los familiares el sacrificio por la patria y un ideal de valores que van unidos a la responsabilidad, obligación moral que se asocia, entre otras cosas, con la idea de cumplir con la palabra dada (Pitt-Rivers, 1979:31). De esta manera, muchos familiares rescatan el hecho de que los caídos hayan cumplido con la palabra dada al jurar la bandera en el Servicio Militar:

"Fue que la situación lo movilizó ¿Por qué? Por los valores de él. Entonces ¿cómo volvía él con sus compañeros que hubieran muerto y todo eso? ¿Se iba a presentar en un aula a hablar de la patria, de San Martín y Belgrano?" (Delmira, madre de un caído en Malvinas, 03-09-09).

Lo que Delmira nos quiere destacar, junto con otros familiares, son los valores de responsabilidad social que relacionan los principios morales del caído en Malvinas con los de San Martín y Belgrano, padres fundadores de la Patria. Activando entonces los símbolos que ofrece la historia nacional, los familiares construyen un nuevo panteón de héroes, y una memoria sobre la guerra, que reclama "no olvidar" los actos heroicos de los soldados.

Lo que resalta de la categoría nativa de héroe nacional es el acto sacrificial. Fueron los griegos quienes nos dieron el legado de asimilar la angustia de la muerte a través del sentido heroico y el orgullo hacia los hombres sacrificados en defensa de la patria (Ferrer: 2006:43) ${ }^{14}$. La importancia que este pueblo le daba a la guerra y al heroísmo en la batalla, fue expresado en la construcción de monumentos y epitafios ("Murió por la patria") a través de los cuales los héroes eran inmortalizados (Ferrer, ibíd.).

Con la misma idea, podemos interpretar de las narrativas de los familiares, específicamente en los casos en los que las madres remarcan su resistencia a que sus hijos fueran a la guerra, que la decisión y voluntad de sus hijos hace que los caídos en Malvinas sean al mismo tiempo víctimas y personas sacrificiales, ya que ellas mismas son quienes ofrecen su vida en consagración a la patria. Si bien son los mismos familiares que se refieren a la experiencia de los héroes como un acto sacrificial, podemos retomar los aportes de Henri Hubert y Marcel Mauss acerca de la naturaleza del sacrificio. Según los autores, el objeto sacrificado, la víctima, pasa del dominio común al religioso, ya que es consagrado, y se modifica el estado de la persona que lo cumple o de alguno de los objetos en los que se interesa (1946: 77). De esta manera, estamos hablando tanto de lo que los autores Ilaman sacrificios personales, como sacrificios objetivos (Ibíd.). En el primer caso, el sacrificio concierne directamente a la persona sacrificante; en el segundo, el efecto principal del rito, recae sobre la cosa que se tiene por objeto a modificar. En el caso de los héroes de Familiares de Malvinas, yo los Ilamé sacrificios voluntarios, ya que, como habíamos

\footnotetext{
14 Así también dice Eulalio Ferrer, Grecia creó prácticamente la mayoría de los rituales fúnebres practicados por los occidentales, como velar al muerto, escoltarlo con coronas florales, y enterrarlos en cementerios (2003:39).
}

señalado, es la misma persona sacrificante la que se ofrece como víctima:

"Y bueno después el llanto de mi mamá, mi hermano tuvo que sentarse y explicarle de que él no se podía quedar acá cuando sus compañeros se estaban yendo y que el... iba a cumplir con lo que había jurado, el había jurado su bandera hasta dar la vida" (María Fernanda, hermana de un caído en Malvinas, 26-06-07).

A través del acto sacrificial, el caído Malvinas, no pasa al mundo de los muertos "comunes" sino, al mundo de los "muertos héroes", como lo hicieran otros próceres argentinos. Esto es así no sólo por el sentido heroico que le dan a las pérdidas de sus seres queridos, sino también porque como hemos visto los caídos en Malvinas como los caídos en las guerra en general, no entran en la categoría de muerte en donde es posible desarrollar los rituales pautados de separación, despedida y agregación al mundo de los muertos ${ }^{15}$.

Para que el sacrifico desde su punto de vista no sea inútil, ya que la guerra se perdió, es fundamental que el intercambio contractual que supone la idea de sacrificio sea obtener cierta soberanía de las Islas a través de los cuerpos que yacen en el cementerio. Así, a partir de la guerra las Islas Malvinas comenzaron a ocupar un lugar primordial en la cartografía simbólica de los familiares. De esta manera, mediante la idea de sacrificio voluntario, y la metáfora orgánica que asocia al cuerpo y la "sangre derramada" con la tierra, los muertos se entienden como metonimia de la tierra. Esta operación simbólica hace que los familiares entiendan a los muertos como si fuesen recursos nacionales para producir soberanía. De ahí el proceso de nacionalización de las muertes que permite la transformación del hombre en héroe, y del héroe en protector de la Patria (panizo, 2011:175). Así, Familiares de Malvinas da sentido a las pérdidas de sus seres queridos a través de un marco simbólico de interpretación que entiende a las muertes como heroicas, nacionales y sagradas.

Pero el marco interpretativo del grupo está enmarcado a la vez, en un contexto histórico-social compartido, por cuanto los familiares recurrieron, en palabras de Alejandro Grimson, a las "configuraciones culturales sedimentadas históricamente" (2007:29). Por un lado, la tradición de guerra ofrece recursos para enfrentarse a la muerte. Como ya he referido, las interpretaciones de los familiares se insertan dentro de un contexto histórico mundial de entendimiento de la muerte en la guerra, en donde a través de estatuas y monumentos de combina el culto a los muertos con el sentimiento nacional. Por otro lado, los familiares dan sentido a las pérdidas tomando recursos que propone la historia nacional. Así recurrieron

\footnotetext{
15 Van Gennep (2008), haciendo referencia a los procesos sociales por los cuales un individuo pasa cuando atraviesa un cambio de estatus o posición social, propone el modelo de los ritos de paso, los cuales divide en fases de separación, de transición y de agregación.
} 
a la Virgen de Luján, como icono de identidad principal, y también a la bandera, los próceres y héroes argentinos, como forma de pensar la identidad y unidad nacional. Las decisiones del Estado a través de normativas y leyes acerca de las denominaciones de las muertes, como la de héroe de guerra, actuaron también como dispositivos que dispusieron de un tipo de fondo social de interpretación de las muertes. Así, Familiares de Malvinas realizan diferentes prácticas oficiales o privadas, públicas o domésticas, como las misas, los bustos, la realización monumento, etc., a través de las cuales representan y experimentan la muerte de los caídos de una forma particular. A estos rituales, los denominé escenarios de la muerte heroica (Panizo 2011:134) retomando los aportes de Turner (1987) acerca de la perspectiva de la performance. Según el autor, el ritual es una performance, una secuencia de actos simbólicos de carácter transformador en donde los sujetos se presentan y revelan a sí mismos, por medio de una escenificación que tiene principio y fin. Así, en una puesta en escena sobre las experiencias de los familiares relativas a las pérdidas de sus seres amados en la Guerra, ellos experimentan los cambios en los estatus y categorías sociales, que son comunicadas a un público particular. En este articulo analizaremos específicamente el descubrimiento del busto del soldado Araujo desde el punto de vista de la performance, ya que nos permite ver de qué manera estamos refiriéndonos a experiencias vivenciadas en escenarios determinados, a través de los cuales es habitada la muerte de una persona, y significada de una manera particular, tal como sucede en la performance de un velatorio tradicional. De esta manera, el ritual que analizaremos a continuación, será entendido como un espacio simbólico de dramatización de la muerte y de reproducción de lazos familiares o de transformación existencial, en un escenario social particular. Por otro lado, veremos de qué manera, el ritual expresa un marco interpretativo particular, en donde la construcción del busto, como forma rememorar la muerte en la guerra, deviene en un ritual mortuorio en donde entran en escena símbolos de identidad nacional, y se enaltece la figura del héroe que ha sacrificado su vida por la Patria.

\section{El caso de Elbio Eduardo Araujo}

Elbio Eduardo Araujo, fue un soldado conscripto clase 62 de la Compañía del Regimiento de Infantería mecanizada N 7 de la ciudad de La Plata, que murió en la Guerra de Malvinas, en la Batalla de Monte Longdon. Si bien su cuerpo yace en el cementerio de Darwin, no está identificado, por los que sus familiares, Maria Fernanda Araujo (hermana) Elbio Araujo (padre) y María del Carmen Penon (madre) eligieron una que decía Soldado Argentino solo conocido por Dios, como lugar de visita y mediación simbólica para encontrarse con su familiar:

"Mi mamá a mi viejo le decía «no está, no está no está» y le decía... "bueno vamos a encontrar una cruz que diga Soldado Argentino solo conocido por
Dios, elegí esa cruz, la que más te parezca, la que más sientas y ahí le dejamos la placa», le dijo papá a mamá. Ella caminó, caminó, caminó, hasta que dijo «Acá»" (María Fernanda, 26/06/07).

El 2 de Julio del año 2007, su familia logró inaugurar el busto en su homenaje en la plaza Malvinas Argentinas, de Colón, Entre Ríos, lugar de su nacimiento. Este acontecimiento, como observaremos a continuación, cumplió un rol muy especial, al igual que la tumba en el cementerio, como locus de ubicación socio espacial de la muerte.

Como refiere Héctor Cisneros acerca de su hermano, soldado de profesión caído en Malvinas, no se acostumbra a levantar bustos a soldados que no hayan sido personal militar:

"Es un reconocimiento a la memoria de un ciudadano que como profesión (énfasis mío) eligió la vida de soldado y que cumplió con su deber". (Héctor, 25/05/09)

Sin embargo, después de la construcción de diversos bustos de militares caídos en la guerra, como el del hermano de Héctor, la hermana del soldado Araujo comenzó las gestiones en el pueblo natal de su hermano, para que se erija un busto en su honor:

"Me dice Héctor: "no hay ningún busto de soldado". Con más razón, que esto sirva de ejemplo para que se desparrame por toda la Argentina, que haya 649 bustos. Empezamos las gestiones" (María Fernanda, hermana del soldado Araujo caído en Malvinas, 09/10/09).

Después de varias negociaciones con la municipalidad de Colón, la familia Araujo, con el apoyo de Familiares de Malvinas, hizo construir el busto de Elbio, el primer busto a un soldado, inaugurándolo en un acto de carácter fúnebre, en donde diferentes autoridades lo legitimaron la muerte del héroe de Malvinas. Con el acompañamiento de la banda del Batallón de Ingenieros Blindados II de Concepción del Uruguay, se cantó el Himno Nacional, la Marcha de Malvinas y la Marcha de Entre Ríos. Quien inauguró el acto fue Cesar Trejo, el apoderado de la institución Familiares de Malvinas. Luego dijo unas palabras la hermana del soldado, María Fernanda, y por último, el intendente Hugo Marsó:

"Es un sentimiento de toda nuestra sociedad, brindar un máximo de gratitud, por el sacrificio, con el respeto y el reconocimiento debido, junto con el deseo de este Pueblo Colonense de brindarle un homenaje a nuestro Héroe, Elvio Eduardo Araujo Penon y todos los que participaron en este hecho tan trascendental en la vida de todos los argentinos, por los valores que esa epopeya encierra y por los gestos humanos realizados"16.

16 "Homenaje al soldado Eduardo Araujo Penon, caído en las Islas en 1982" en http://www.malvinense.com.ar/smalvi/utj072007/00394. 
Luego, el presidente municipal junto a familiares descubrieron el busto y la placa del soldado, bendecido por el Padre Néstor Toler, dando lugar a las ofrendas florales que realizaron diferentes funcionarios del Estado, Familiares de Malvinas, el Instituto Nacional Juan Manuel de Rosas, representantes del Jefe de Estado Mayor del Ejército y de CECIMER (Centro de ex combatientes de Islas Malvinas de Entre Ríos) y del Instituto Malvinas Patagónicas e Islas del Atlántico Sur. Presenciaron la ceremonia también, alumnos representantes de distintas escuelas públicas de Cólon, ex combatientes de Florencio Varela, Berazategui, Capital Federal y Concepción del Uruguay entre otras localidades, otros familiares del héroe (sobrinos, primos, etc.) y la figura de la Virgen de Luján.

Lo que es significativo es que el acto de inauguración se desarrolló como un ritual mortuorio religioso en donde, como un velatorio, a través y a partir del cuerpo metálico, los familiares "tocaron" y lloraron al muerto" y los amigos y demás presentes, saludaron y se solidarizaron con los dolientes:

"Una ceremonia muy emotiva. Linda, estuvo la ceremonia, muy emotiva. Después, hablaron ellos, se habló tan bien...después, viste, todo el mundo opina y recuerda como fue él, siempre, que fue tan cálido, tan bueno, tan compañero era. Estaba ella, que habló tan bien, Muy acompañados, igracias a Dios! En fin, sentimos que se lo reconoce" (María del Carmen madre del soldado Araujo caído en Malvinas 01/09/09).

Así también, es relevante remarcar que fue llamativa la figura de la Virgen de Luján, que ocupando un lugar privilegiado al lado del busto, y al igual que en todas las actividades públicas organizadas por Familiares de Malvinas, recibió también visitas y gestos de gratitud.

Reflexionando sobre varios casos en los que levantan y derriban estatuas, refiere Katerine Verdery acerca de ellas, que "son personas muertas fundidas en bronce o talladas en piedra", ya que no "solo simbolizan a una persona famosa específica sino que en cierto sentido, son también el cuerpo de esa persona" (1999:5)*. Así, dice la autora, muchas estatuas son tratadas como cuerpos muertos y devienen en lugares de peregrinación o manifiestan resonancia mágica o religiosa (Ibíd.: 12). Entender a la estatua como "el cuerpo muerto fundido en bronce" es altamente significativo cuando ésta es la materialidad a través de la cual se ejercen rituales mortuorios que no pudieron hacerse por la falta del cuerpo. Las percepciones que puede generar la estatua, en este caso el busto del soldado Araujo en el contexto ritual, nos hacen comprender la actitud de su mamá, de querer ir una noche de lluvia con un paraguas a proteger el busto para que no se mojara. Nos permite comprender también la forma en que todo sucedió como si fuese la recreación del velatorio que el soldado no tuvo, en el cual los familiares se acercaron para tocar y acompañar al muerto, y en donde los presentes asistían para dar las condolencias con gestos de gratitud por el sacrificio ofrecido con palabras tales como "Gracias por tu hermano".

Así, al suspender el proceso de descomposición del cuerpo, refiere Verdery (1999) la estatua "altera la temporalidad asociada a la persona" (Ibíd.) * por lo que los familiares lloraron al muerto y los presentes acompañaron a los deudos como si se tratara de una muerte ocurrida recientemente y no veinticinco años atrás:

Las investigaciones antropológicas sobre rituales mortuorios en diversas sociedades junto con mi propia etnografía en velatorios, nos sugieren identificar el cuerpo muerto como un símbolo ritual que se convierte en un factor de acción social, ya que sobre él y alrededor de él se ejercen las prácticas rituales. En el velatorio, con la presencia del cuerpo, se le da lugar al muerto de tener una última permanencia con los vivos y a los vivos una última permanencia con aquél. A éste se le habla, se lo

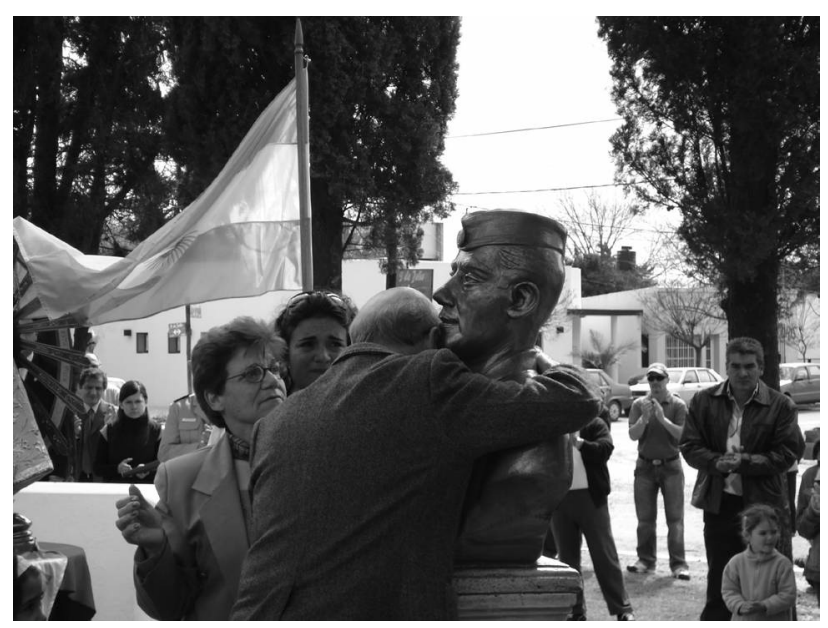

Figura 1. El padre del soldado Araujo expresando su dolor frente al busto de su hijo. Fotografía de Laura Panizo.

Figure 1.The soldier's father expressing his grief against the bust of his son. Picture by Laura Panizo.

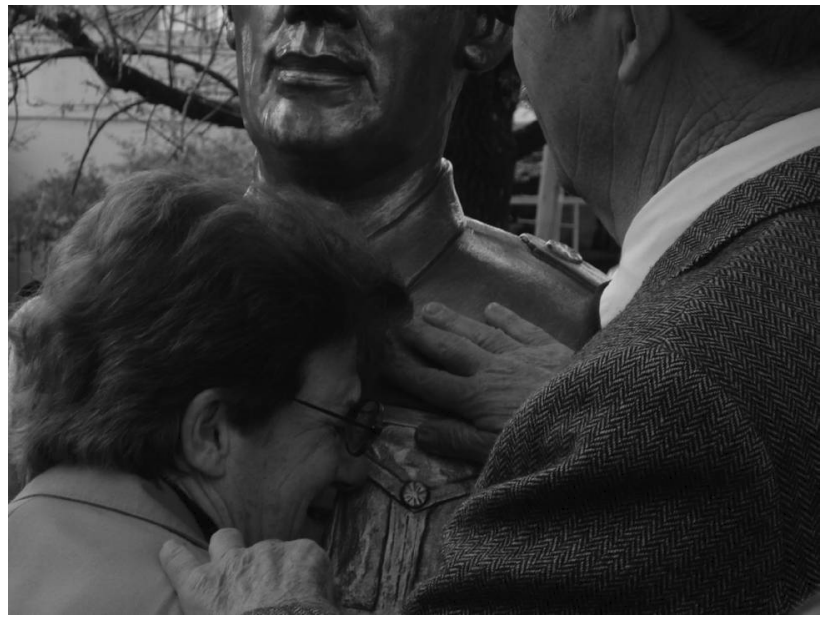

Figura 2. La madre del soldado Araujo expresando su dolor frente al busto de su hijo. Fotografía de Laura Panizo.

Figure 2. The soldier's mother expressing his grief against the bust of his son. Picture by Laura Panizo. 
contempla, se lo llora, acaricia, y despide. La presencia del cuerpo también remite, a las normas y valores que guían a los individuos dentro de la sociedad, ya que especifica claramente la categoría de doliente en la que se encuentra el familiar, persona allegada al muerto que está pasando por una situación especial y que, a partir de entonces, tiene que seguir conductas determinadas. Así, el velatorio reúne a parientes y amigos y se crea entonces un espacio social donde los dolientes se sienten acompañados, "entendidos" y atendidos. Su dolor ante la pérdida es reconocido por parte de la sociedad, ya que la muerte se hace pública y es habitada y compartida socialmente (Panizo, 2011 b). Además de permitir que se generen espacios donde uno se enfrenta a la muerte "en persona", el velatorio da lugar a que el familiar pueda honrar a su fallecido a través de las ofrendas florales. Para ello, además de estas ofrendas, los familiares ofrecen palabras de gratitud para con los muertos, traen anécdotas conmemorables y resaltan las cualidades de las personas.

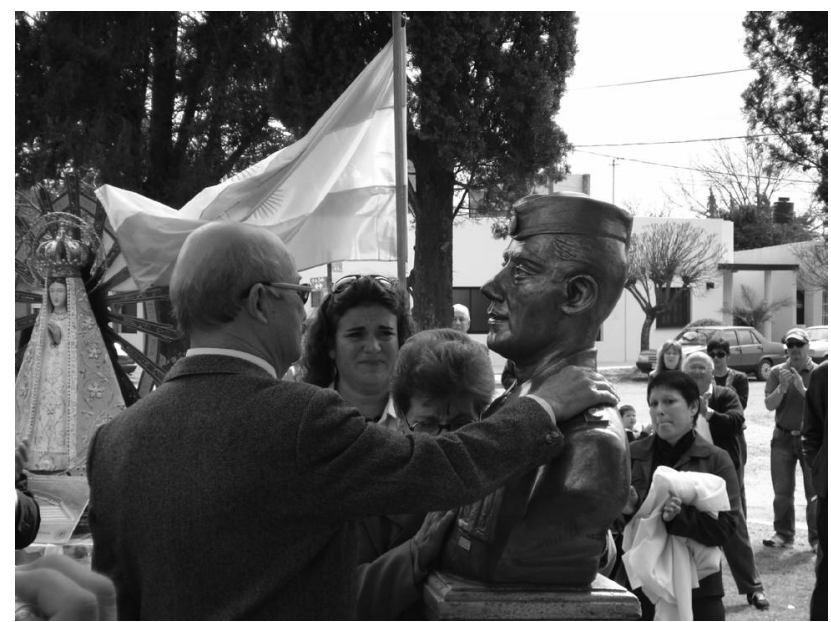

Figura 3. La madre, el padre y la hermana del soldado cerca del busto. Fotografía de Laura Panizo

Figure 3. Mother, father and sister of the soldier around the bust. Picture by Laura Panizo.

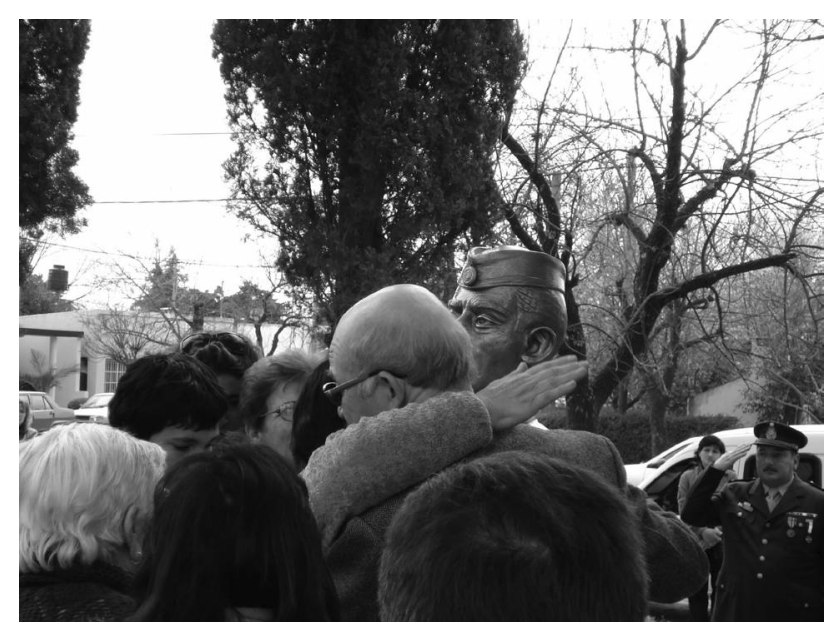

Figura 4. Personas saludando a los deudos. Fotografía de Laura Panizo.

Figure 4. People greeting the mourners. Picture by Laura Panizo.
Como vemos, el descubrimiento del busto devino en un ritual mortuorio que al igual que en el velatorio permitió que los familiares y presentes presten atención al muerto, a través del busto, y puedan rendir sus honores con ofrendas florales y palabras de gratitud. Se creó también, un espacio de solidaridad donde los deudos se sintieron acompañados y comprendidos por el resto de los presentes. Si bien la familia Araujo ha participado y participa de otras prácticas organizadas por la comisión en las que los familiares les rinden honores a los muertos de la guerra ${ }^{17}$, esta fue la primera vez que rindieron culto específicamente a su familiar en un espacio social en la participaron también otros familiares, amigos, ex combatientes, y autoridades locales que dieron legitimidad al acontecimiento. De esta manera, un ritual mortuorio que no había tenido lugar por la falta del cuerpo, sucedió a partir del descubrimiento del busto, lo que permitió el reconocimiento social de la muerte y de la condición del soldado Araujo, de Héroe de la Patria.

Pero el busto para los familiares del soldado, no solo significó el reconocimiento oficial a un héroe, sino el de un soldado conscripto, que merece un reconocimiento diferente en tanto tal, a diferencia del personal de cuadro, ya que no fue a Malvinas por su profesión:

"Para mi, el orgullo más grande es que fue soldado (negrita agregada). A veces, pasa que escuchás: "viste, era oficial o suboficial", pero con orgullo y, "tu hermano era soldado", te suele pasar, también. Entonces, me río y digo: "bueno, si vamos a poner, en la balanza del heroísmo, jflaco! mi hermano era un soldado raso, raso, soldado de Servicio Militar obligatorio". Mi hermano fue lleno de orgullo, entonces, no me vengas con que tu hermano era soldado, despectivo" (María Fernanda, hermana del soldado Araujo caído en Malvinas 03/04/09).

"El sentimiento nuestro es... es ese orgullo que uno tiene de tener ese hijo héroe. Un reconocimiento al héroe. Luchamos para conseguirlo y lo tenemos, fue el primer busto de un soldado. Es un orgullo tan grande para uno, por supuesto, como para cualquier padre, madre. Al verlo, averiguan y se enteran, para que no se olviden" (María del Carmen madre del soldado Araujo caído en Malvinas, 01/09/09).

"Pasa mucha gente por ahí. Y, despacito, la gente fue reconociendo, dándose cuenta de quién fue el soldado. Único, el primer busto en la República Argentina a un soldado (Elbio, padre del soldado Araujo caído en Malvinas 01/09/09).

A la vez, en la performance de este ritual, no sólo se estaba vivenciando la muerte de un caído, sino que

17 Entre estas prácticas encontramos las misas del 2 de abril, las peregrinaciones con la Virgen de Lujan, las muestras itinerantes “Islas de la Memoria", y la participación en la inauguración del cenotafio en el cementerio de Darwin, Isla Soledad. Para profundizar sobre estas prácticas ver Panizo 2011:128-173). 
entraban en escena símbolos de identidad nacional hegemónica, como la participación del ejército, la Virgen, y banderas argentinas. Los discursos de los familiares y funcionarios del Estado resaltaban también la dimensión política del ritual: no sólo atendían a la pérdida de un pariente o un amigo, sino a la de un ciudadano, que dio su vida representando a la comunidad nacional. De esta manera, el ritual expresa el marco interpretativo del grupo, y refuerza públicamente no sólo el vínculo de los familiares con su muerto, sino el de éste y ellos con la Patria y la causa nacional.

\section{Palabras finales}

En este artículo observamos de que manera los familiares dan sentido a las pérdidas a través de un marco interpretativo que entiende a la muertes en la guerra en tanto acontecimiento histórico de importancia nacional. A través del análisis del descubrimiento del busto del Soldado Araujo, observaremos una práctica ritual que no solo enaltece la figura del caído como héroe nacional, sino que habilitan que la muerte se pueda habitar en espacios de atención social. Así esta puesta en escena, no sólo actúa como un canal de comunicación a través del cual los deudos le expresan al resto de la sociedad sus experiencias y los sentidos dados a la muerte en la guerra, sino que también, para los familiares involucrados, son prácticas transformadores en cuanto a la reflexividad que proponen sobre la condición social de los muertos. Concluyendo, el primer busto que se realizó a un soldado de Malvinas, dio lugar no sólo a la posibilidad de un ritual mortuorio por la falta del cuerpo sino a la conquista de un espacio público para simbolizar las experiencias vividas en la guerra, y las representaciones de responsabilidad ciudadana e identidad nacional.

Ciudad Autónoma de Buenos Aires, 10 de Febrero de 2012

\section{Bibliografía}

Álvarez, S. 2001. "Enterrando heróis, patriarcas, suicidas e traidores: solidariedade e ostracismo nos andes colombianos". MANA, 7(2):35-55.

Ariès, P. 1999. El hombre ante la muerte. Grupo Santillana de Ediciones, Madrid.

Carozzi, M. 1998. El concepto de marco interpretativo en el estudio de movimientos religiosos. Sociedad y Religión 16/17:33-51.

Ferrer, E. 2003. El lenguaje de la inmortalidad. México. Fondo de Cultura Económica.

Grant, S. 2004. Patriot Graves: American National Identity and the Civil War Deae. American Nineteenth Century History, 5(3):74-100.

Goffman, E. 1974. Frame análisis: An essay on the organization of experience. New York. Harper Colophon Books.

Guber, R. 2001. ¿Por qué Malvinas? De la causa nacional a la guerra absurda. Fondo de Cultura Económica, Buenos Aires.

Guber, R. 2004. De chicos a veteranos. Memorias argentinas de la guerra de Malvinas. Antropofagia, Argentina.

Halbwachs, M. 2005. "Memoria individual y memoria colectiva". Estudios 16:163-187.

Halbwachs, M. 2004. La memoria Colectiva. Traducción de Inés Sancho-Arroyo. Zaragoza, Prensas Universitarias de Zaragoza.

Jelin, E. 2002. Los trabajos de la memoria. Siglo XXI, Madrid.

Kohan, M. 2005. Narrar a San Martín. Adriana Hidalgo editora, Buenos Aires.

Le Goff J. 1991. El orden de la memoria. Paidós, Barcelona.

Lorenz, F. 2006. Las guerras por Malvinas. Editorial Edhasa, Buenos Aires.

Panizo, L. 2011a. Donde están nuestros muertos: experiencias rituales de familiares de desaparecidos de la última dictadura militar en la Argentina y caídos en la Guerra de Malvinas. Facultad de Filosofía y Letras. Universidad de Buenos Aires. Argentina. Tesis de doctorado. Facultad de Filosofia y Letras, UBA.

Panizo. L. 2009. Muerte, desaparición y memoria: el caso de los desaparecidos de la última dictadura militar en Argentina. Historia, Antropología y Fuentes Orales. 42: 71-84.

Panizo, L. 2011 b. Cuerpo, velatorio y performance. Revista Tán@to's. Sociedad Española e Internacional de Tanatología. 13:24-35

Pollak, M. 2006. Memoria, olvido, silencio. Ludmila da Silva Catela (comp.). Al Margen. La Plata.

Pitt-Rivers, J.1979. Antropología del Honor. Crítica, Barcelona,

Stephens, J. 2007. Memory, Commemoration and the Meaning of a Suburban War Memorial. Journal of Material Culture, $12: 241-261$.

Turner, V. 1987. The Anthropology of Performance. PAJ Publications, New York

van Gennep, A. 2008. Los ritos de paso. Alianza Editorial, Madrid.

Verdery, K. 1999. The political lives of dead bodies: reburial and postcocialist change Columbia Universiyy Press, United States of America. 